\title{
Co-Transplantation of Skin-Derived Precursors and Collagen Sponge Facilitates Diabetic Wound Healing by Promoting Local Vascular Regeneration
}

\author{
Tingyu Ke Mei Yang $^{\mathrm{a}}$ Duo Mao ${ }^{\mathrm{b}}$ Meifeng Zhu ${ }^{\mathrm{b}}$ Yongzhe Che ${ }^{\mathrm{b}}$ Deling Kong ${ }^{\mathrm{b}, \mathrm{c}}$ \\ Chen $\mathrm{Li}^{\mathrm{c}}$ \\ aDepartment of Endocrinology, The Second Affiliated Hospital of Kunming Medical University,

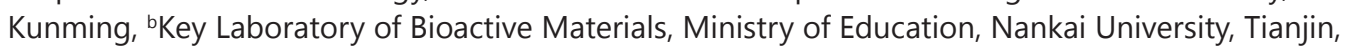 \\ 'Tianjin Key Laboratory of Biomaterial Research, Institute of Biomedical Engineering, Chinese Academy \\ of Medical Science, Tianjin, China
}

\section{Key Words}

Skin-derived precursors • Diabetes • Wound healing • Collagen sponge

\begin{abstract}
Background/Aims: Impaired diabetes wound healing can often lead to serious complications and remains a major health concern due to the lack of effective therapeutic approaches. Compromised angiogenesis, disrupted growth factor and cytokine activity are all attributable to diabetic wound healing impairment. The skin-derived precursors (SKPs) have been shown to differentiate into vascular and nerve cells, both of which are crucial components for wound repair. Given their easy accessibility and multipotency, the SKPs were proposed as an ideal therapeutic candidate for diabetic wound healing. Since the efficacy of cell therapy is limited by poor cell survival, collagen sponge was employed for better SKPs delivery. Methods: SKPs were isolated and transplanted directly to the wound areas of diabetic mice in the absence and presence of collagen sponge. The effects of SKPs and/or collagen sponge on diabetic wound healing were examined histologically as well as immunostaining of isolectin and $\alpha$-SMA. Mechanisms via which the SKPs facilitate wound healing were then investigated by transplanting SKPs that have been pre-labelled with a fluorescence dye, Dil. Expression patterns of Dil and an SKP marker, nestin, was also examined. Results and Conclusion: Accelerated wound healing and enhanced local capillary regeneration could be observed 14 days after skin ablation from both SKPs and collagen sponge co-transplanted and collagen sponge only groups. Subsequent analyses further revealed superior pro-angiogenic effects from the SKP and collagen sponge co-delivered group, which are mainly attributable to in vivo transdifferentation and paracrine signalling of the SKPS.




\section{Cellular Physiology Cell Physiol Biochem 2015;37:1725-1737 and Biochemistry Published online: November 09, 2015 www.karger.com/cpb \\ Ke et al.: Skin-Derived Precursors Promote Angiogenesis in Diabetic Wound Healing}

\section{Introduction}

Diabetes is a heterogeneous metabolic disorder characterised by sustained hyperglycaemia as a result of insufficient insulin secretion. Epithelialisation defect is prevalent in diabetic patients, with impaired cutaneous wound healing being one of the most common diabetic complications that often leads to diabetic ulcers, epithelial erosion, chronic lesions, and in some cases, lower extremities amputation. Wound healing is a complex remodelling process composed of multiple stages including coagulation, inflammation, granulation and angiogenesis [1]. Several cytokines and growth factors are involved in mediating each stage, although this paracrine regulation is often compromised in diabetes. Progenitor cell mobilisation is also impaired in diabetes, due to reduced local neoangiogenesis and blood flow at the wound area. In addition, long-term poor glycaemic control, chronic peripheral vasculopathy, neuropathy and increased susceptibility to infection have all been proposed to exacerbate skin tissue damage, mainly by attenuating cell migration, proliferation and extracellular matrix deposition [2]. Anti-microbial therapy and surgical debridement are some commonly used therapeutic interventions against diabetic lesion and infections [3]. Other approaches such as advanced moist wound therapy, negative pressure wound therapy, hyperbaric oxygen therapy as well as skin transplantation have also been assessed for its efficacy against diabetic wounds, but the clinical outcomes are less than satisfactory $[1,3,4]$.

In the past decade, the potential of stem cell-based therapy in tissue regeneration has been extensively investigated [5-9]. The skin-derived precursors (SKPs) are multi-lineage precursor cells derived from human and rodent dermis. They could be maintained as selfrenewing spheres in vitro and differentiate into functional cells of neural and mesodermal origins [10-12]. Owing to its multipotency and easy accessibility, SKPs have been considered an ideal candidate for cell therapy. Indeed, protective impact of the SKPs in spinal cord injury was initially reported since they were able to promote neuron regeneration and remyelination axon by differentiating into functional myelinating Schwann cells [13, 14]. In addition, directed generation of islet-like insulin-producing cells from SKPs was observed in vitro $[15,16]$, and differentiation of the SKPs into cells that resembled native chondrocytes and osteocytes morphologically and functionally was also demonstrated which facilitated bone reconstruction in vivo [17].

More recently, the SKPs have been proposed to promote skin regenerating during wound healing [18]. The follicular dermal papillae, one niche of the endogenous SKPs, have long been known to play a part in skin turnover and wound healing [19]. Earlier studies have already shown that transplantation of nestin ${ }^{+}$hair follicular stem cells could facilitate functional restoration of the nerves [13] and formation of capillary networks [20], both processes are required in complete skin wound healing. Considering that the SKPs 1) exhibit multi-lineage property towards neurons and smooth muscle cells, and 2) express nestin, a neural precursor marker, it has been suggested that the SKPs were, at least in part, facilitative in nerve regeneration and angiogenesis following tissue injury. A later study using SKPs isolated from adult foreskin demonstrated TGF- $\beta 1$ /TGF- $\beta 3$-induced differentiation into smooth muscle cells, which functionally resemble vascular smooth muscle cells. More importantly, these SKPs-derived vascular smooth muscle cells could form contractile capillaries in vivo that were able to integrate with the native capillary vasculature [21]. Given the importance of capillary regeneration in wound healing and the detrimental impact of diabetes on microcirculation, we sought to investigate the therapeutic potential of SKPs transplantation in diabetic wound healing.

However, one problem of stem cell-based therapies is poor survival of the precursor cells following transplantation as a result of local ischaemia, inflammation and hypoxia [7, $22,23]$. In order to improve cell viability and survival, natural and synthetic biomaterial scaffolds have been employed for stem cell culture and delivery [24, 25]. Collagen sponge is composed of the extracellular matrix protein, collagen, and provides mechanical support as well as biological function after implantation [26-28]. Indeed, collagen sponge has been shown to promote mesenchymal stem cell differentiation and epithelial cell proliferation 


\section{Cellular Physiology Cell Physiol Biochem 2015;37:1725-1737 \\ \begin{tabular}{ll|l}
\cline { 2 - 2 } and Biochemistry $10.1159 / 000438537$ & $\begin{array}{l}\text { ( ) 2015 The Author(s). Published by S. Karger AG, Basel } \\
\text { www.karger.com/cpb }\end{array}$ \\
\cline { 1 - 3 }
\end{tabular} \\ Ke et al.: Skin-Derived Precursors Promote Angiogenesis in Diabetic Wound Healing}

[28]. Collagen-based scaffolds have been used clinically for decades in tissue engineering, such as bone, cartilage, and ligament $[29,30]$. Cutaneous wound dressing was also developed using collagen sponge as a result of its coagulant and pro-proliferative properties [31-33]. Given the beneficial impact of collagen sponge in wound closure and stem cell delivery, SKPs were co-transplanted with a collagen sponge scaffold and the therapeutic potential of the collagen sponge-delivered SKPs on post-injury skin regeneration and wound site vascular reformation was evaluated in diabetic mice.

\section{Materials and Methods}

\section{Materials}

Collagen sponge was given courtesy of ZH-BIO (Yantai, China). All cell culture reagents were obtained from Gibco (Beijing, China). Collagenase V, DAPI, streptozocin, MTT and Masson trichrome staining kit were purchased from Sigma-Aldrich (Dorset, UK). Dil was obtained from Molecular Probes (Shanghai, China). Mayer's haematoxylin was purchased from AbCam (Hangzhou, China) and eosin Y was from Solarbio (Beijing, China). Anti-Nestin and anti- $\alpha$-SMA antibodies were purchased from Santa Cruz Biotechnology (Shanghai, China). Rabbit anti-human vWF antibody was obtained from Dako (Shanghai, China). Alexa 486-conjugated isolectin-B4 and FITC-conjugated secondary antibody were from Invitrogen (Beijing, China). All animals were purchased from the Laboratory Animal Centre of the Academy of Military Medical Sciences (Beijing, China). Procedures performed in the present study were in compliance with the regulations of the Tianjin Committee of Use and Care of Laboratory Animals and the overall project protocol was approved by the Animal Ethics Committee of the Nankai University.

\section{Animals}

Male C57BL/6J mice (3-4 weeks old, 12-14g body weight) were put on a high fat diet (55\% calories from fat) for 5 weeks. At week 6, 1\% streptozocin (STZ, $85 \mathrm{mg} / \mathrm{kg}$ body weight) were administrated twice with two days in between. Fasting plasma glucose levels were measured on day 7 and 14 after the initial injection of STZ. Mice with fasting plasma glucose levels over $16.7 \mathrm{mmol} / \mathrm{L}$ were considered diabetic.

\section{Skin excision wound surgery}

Animals were anesthetised by intraperitoneal (i.p.) injections of $4 \%$ chloral hydrate solution (350 mg/ $\mathrm{kg}$ body weight). The dorsum of each mouse was shaved and sterilised before a full-thickness skin wound ( $8 \mathrm{~mm}$ in diameter) was created using a biopsy punch to ensure standardised wounds. Wound closure was monitored with a digital camera. Photographs were taken immediately after the surgery (day 0 ) and on day 7 and 14 afterwards with a ruler photographed underneath each wounds. The areas of open wounds were calculated using Image-pro plus 6.0 (IPP) software. Open wound size was defined as non-epithelialised area and measured using a vernier caliper.

\section{Isolation of SKPS}

To obtain SKPs, C57BL/6J mice (male, 3-4 weeks old) was shaved, the dorsal skin was cut and washed with sterilised PBS before being macerated and digested with $1 \mathrm{mg} / \mathrm{mL}$ Dispase at $4^{\circ} \mathrm{C}$ overnight. The dermis was then digested with $0.5 \mathrm{mg} / \mathrm{mL}$ collagenase in DMEM/F12 for 1 hour at $37^{\circ} \mathrm{C}$. Cell suspension was passed through a $40 \mu \mathrm{m}$ cell strainer before washed with DMEM twice. Cell pellet was resuspended in DMEM/F12 media supplemented with $20 \%$ foetal bovine serum (FBS), penicillin $(100 \mathrm{unit} / \mathrm{mL})$, streptomycin $(100 \mu \mathrm{g} /$ $\mathrm{mL})$, non-essential amino acid $(0.1 \mathrm{mmol} / \mathrm{L})$ and L-glutamine $(2 \mathrm{mmol} / \mathrm{L})$ and plated in a $100 \mathrm{~mm}$ plate to be maintained under standard culturing condition $\left(37^{\circ} \mathrm{C}, 95 \%\right.$ air $\left./ 5 \% \mathrm{CO}_{2}\right)$.

\section{Co-culturing of SKPs and collagen sponge}

The collagen sponge was first prepared for scanned electron microscopy (SEM) according to standard procedure [34]. After characterisation, the material was cut into discs ( $8 \mathrm{~mm}$ in diameter) and sterilised with Co60 (20K). The collagen sponge discs were then placed into designated wells of a 48 -well tissue culture plate and maintained in $300 \mu \mathrm{l} \mathrm{SKP}$ culture media per disc at $37^{\circ} \mathrm{C}$ overnight. For SKP seeding, approximately $1 \times 10^{5} \mathrm{SKPs}$ in $20-30 \mu \mathrm{l}$ media were seeded to each disc and maintained for 4 hours at $37^{\circ} \mathrm{C}$. 


\section{Cellular Physiology Cell Physiol Biochem 2015;37:1725-1737

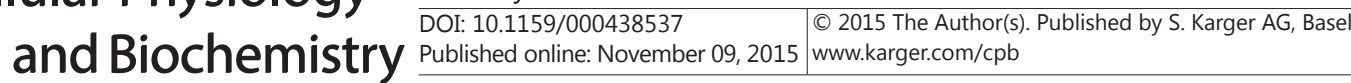 \\ Ke et al.: Skin-Derived Precursors Promote Angiogenesis in Diabetic Wound Healing}

$300 \mu \mathrm{l}$ culture media was added into each well afterwards and the plate was kept under standard culture condition for further experimental use.

To assess the viability of SKPs after co-culturing, MTT assay was carried out as previously detailed [7]. Briefly, $25 \mu \mathrm{l}$ MTT solution ( $5 \mathrm{mg} / \mathrm{mL}$ ) was added into each sample well. After 4 hours at $37^{\circ} \mathrm{C}$, the MTT solution was aspirated off. Each collagen sponge was cut into quarters followed by addition of $300 \mu \mathrm{l}$ DMSO to wash out the SKPs. $50 \mu \mathrm{l}$ of the cell suspension of each sample was then transferred to a 96-well plate and the optical density (OD) value was measured using a spectrophotometer at a wavelength of $490 \mathrm{~nm}$.

Transplantation of SKPs with collagen sponge

Isolated SKPs were maintained in culture at $37^{\circ} \mathrm{C}$ and labelled with fluorescent dye, Dil $(3 \mu \mathrm{g} / \mathrm{mL})$, for 8 min. Excessive dye was rinsed off with sterilised PBS. The SKPs were counted and seeded onto collagen sponge discs as detailed above and transplanted directly to the wound area immediately after skin ablation.

\section{Histomorphological and immunofluorescence analysis}

Animals were sacrificed on day 7 and 14 following initial skin wound. The excision areas $\left(\sim 1 \mathrm{~cm}^{2}\right)$ were obtained, fixed in $4 \%$ paraformaldehyde, paraffin embedded and consecutive sections of $6 \mu \mathrm{m}$ were cut onto microscope slides. Haematoxylin \& eosin (H\&E) staining was employed to examine epithelial thickness. For immunofluorescence staining, the sections were dewaxed, rehydrated and permeabilised with $0.1 \%(v / v)$ Triton $\times-100$ in TBS followed by antigen retrieval using Tris-EDTA. The sections were then incubated at $4^{\circ} \mathrm{C}$ overnight with FITC-conjugated antibodies against $\alpha$-SMA (1/100 dilution), nestin (1/100 dilution), von Willebrand factor (vWF; $1 / 200$ dilution) and isolectin (1/70 dilution). DAPI was used for nuclei counterstaining. The immunofluorescence was examined under a con-focal microscope (Leica, Germany) and quantified using Image J software (Wayne Rasband, National Institute of Health, USA).

\section{Statistical analysis}

Numerical data are expressed as means \pm standard deviation of multiple independent sets of experiments. Differences between two groups were analysed by Student's $t$-test. One-way ANOVA was used for multiple comparisons or Tamhane's T3 test, as appropriate. Values of $p<0.05$ were considered statistically significant.

\section{Results}

\section{Effect of collagen sponge co-culturing on in vitro SKPs proliferation}

To evaluate the potential benefit of collagen sponge on in vivo SKPs delivery, cell viability test was initially employed to assess the effect of collagen sponge on SKPs proliferation and survival in vitro. Thus, the SKPs were cultured in the presence and absence of collagen sponge for 5 days. MTT assays were carried out on Day 1, 3 and 5 for the SKPs-collagen co-cultured group and the parallel SKPs-only group. The collagen discs were also harvested at the end of Day 1, 3 and 5 for electron microscopy. As shown in Fig. 1a, fibroblast-like SKPs could be observed within the collagen sponge scaffold. Average cell density visibly increased as the culturing period extended. Consistently, results from MTT assays also showed elevated SKPs proliferation from the SKPs-collagen co-cultured group (162 $\pm 12 \%$ and $178 \pm 11 \%$ on Day 1 and 3 respectively, data shown as percentage over Day $1, p<0.01$ vs Day 1) and the SKPsonly control group $(146 \pm 11 \%$ and $169 \pm 6 \%$ at Day 1 and 3 respectively, data shown as percentage over Day 1, $p<0.01$ vs Day 1; Fig. 1b).

\section{SKPs and collagen sponge transplantation accelerates cutaneous wound healing in T2D} mice

Multiple studies have reported impaired cutaneous wound healing in diabetic animals $[1,3,35,36]$. To investigate the impact of SKPs and collagen sponge on wound healing in diabetes, T2D mice were subjected to dorsal skin ablation and treated with co-transplantation of SKPs and collagen sponge (SKPs + collagen) or collagen sponge without SKPs (collagen only). Mice from the control group were administered with saline and the healing processes 


\section{Cellular Physiology \\ Cell Physiol Biochem 2015;37:1725-1737 \\ \begin{tabular}{l|l}
\hline DOI: $10.1159 / 000438537$ & C 2015 The Author(s). Published by S. Karger AG, Basel
\end{tabular} and Biochemistry Published online: November 09, 2015 www.karger.com/cpb

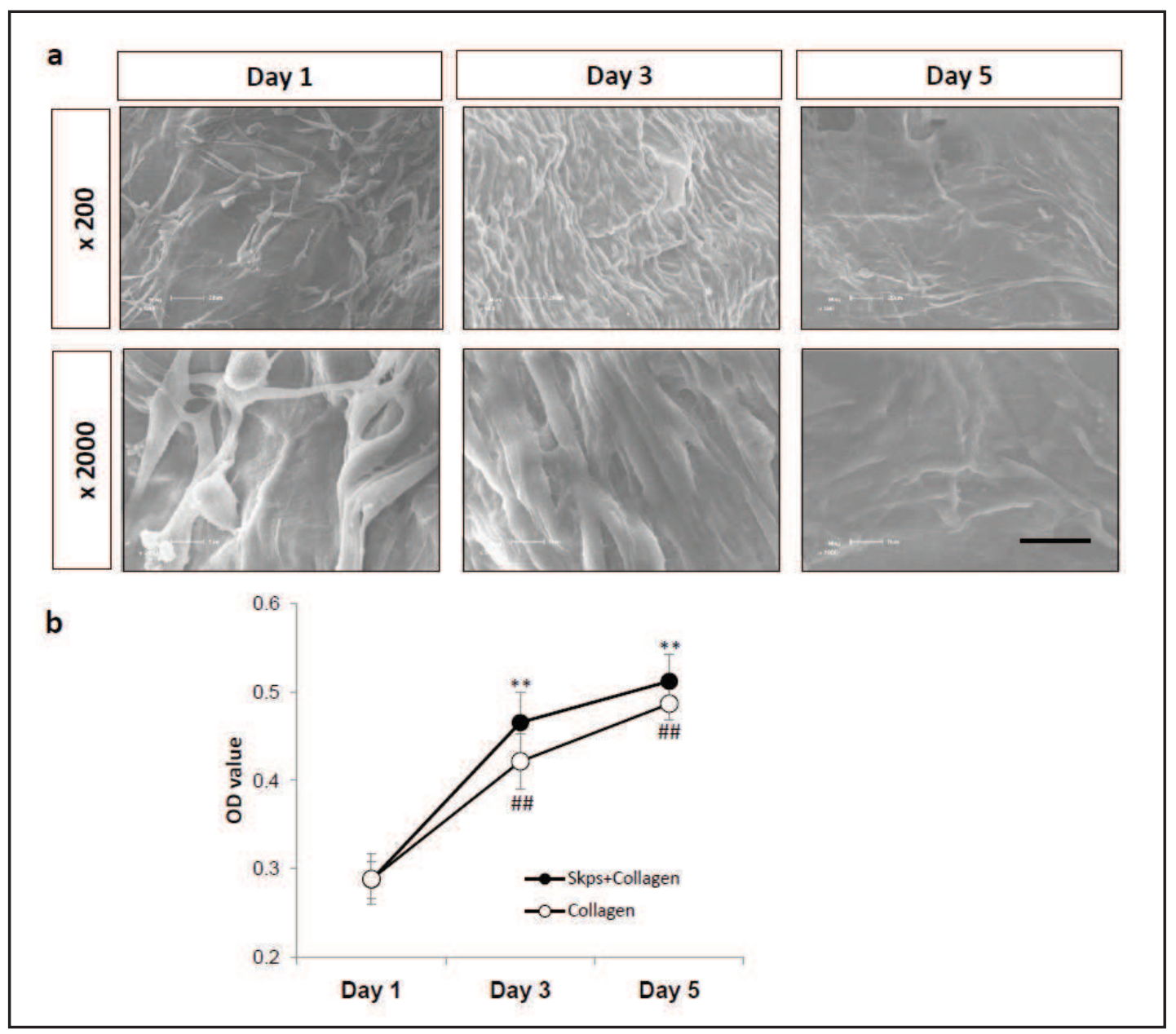

Fig. 1. Co-culture of the SKPs with collagen sponge scaffold. (a) Transmission electron micrograph of collagen sponge scaffolds and SKPs in culture after Day 1, 3 and 5. Scale bar $=50 \mu \mathrm{m}$. (b) MTT assay of SKPs that were maintained in culture in the presence (closed circle) and absence (open circle) of collagen sponge at Day 1, 3 and 5. Significant increase in cell growth is shown in both groups in comparison to SKPs harvested on Day 1. Images are representative of 6 independent experiments. Results are means of 3 independent experiments and data are shown as mean \pm standard errors. ${ }^{* *} p<0.01$ compared to Day 1 of SKPs + collagen group; \#\#p $<0.01$ compared to Day 1 of the SKPs only control group.

were monitored over a 14-day period. Areas of skin wounds were photographed and measured on Day 0, 7 and 14. Representative images are shown in Fig. 2a. Mice treated with either collagen only or SKPs-collagen exhibited significantly reduced residual wound areas compared to the control group on Day 7 (SKPs + collagen: $51 \pm 14 \%$ over Day $0, p<0.01 v s$ control; Collagen only: $54 \pm 18 \%$ over Day $0, p<0.05$ vs control) and Day 14 (SKPs + collagen: $12 \pm 4 \%$ over Day $0, p<0.05 v s$ control; Collagen only: $10 \pm 4 \%$ over Day $0, p<0.05 v s$ control; Fig. 2b).

In addition, wound closure irrespective to areas of initial wounds was also evaluated. As shown in Fig. 2c, both SKPs + collagen and collagen only groups had markedly better wound closure at Day 7 (SKPs + collagen: $49 \pm 14 \%$ wound contraction, $p<0.01$ vs control; Collagen only: $39 \pm 12 \%$ wound contraction, $p<0.05 v s$ control). However, towards the end of the 14day observation period, fibrin crusts were invariably formed in mice from all 3 groups. In the SKPs + collagen and collagen only groups, the crusts had resolved before Day 14 (Fig. 2a), demonstrative of accelerated wound healing compared to the controls. 


\section{Cellular Physiology \\ Cell Physiol Biochem 2015;37:1725-1737

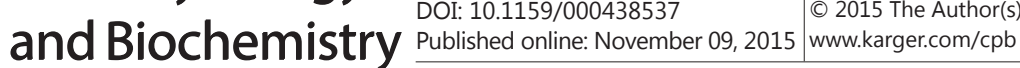 \\ Ke et al.: Skin-Derived Precursors Promote Angiogenesis in Diabetic Wound Healing}

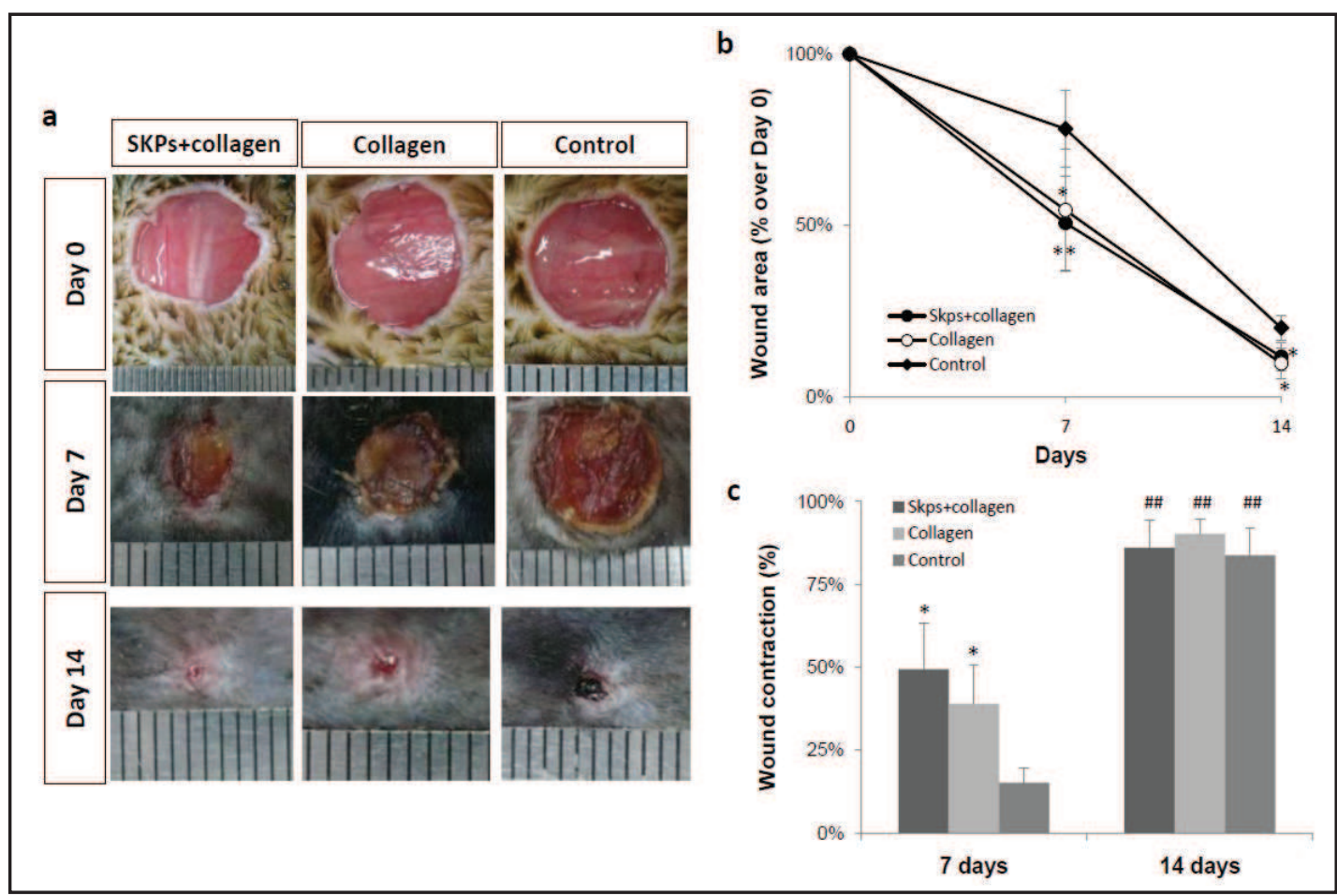

Fig. 2. Effect of collagen sponge-delivered SKPs or collagen sponge only treatment on cutaneous wound closure in T2D mice. (a) Representative microscopic images of cutaneous wounds following treatment of collagen sponge-delivered SKPs or collagen sponge only. Photographs were taken immediately after skin abrasion (Day 0), on Day 7 and Day 14. (b) Changes of wound area, expressed as percentage over wound area at Day 0. (c) Rate of wound contraction. Images are representative of 6 independent experiments. Mice with skin abrasion but no treatment at the wound area were considered as controls. Results are means of 6 independent experiments and data are shown as mean \pm standard errors. ${ }^{*} p<0.05,{ }^{* *} p<0.01$ compared to control group; \#\#p<0.01 compared to Day 7 of respective groups.

\section{SKPs and collagen sponge transplantation enhances reepithelialisation}

Reepithelialisation has long been considered a major event in successful wound healing. To assess the impact of SKPs and collagen sponge on wound closure, dorsal skin surrounding the wound area was collected from diabetic mice. Epidermal reconstitution was examined by H\&E and Masson trichrome staining. Fig. 3a demonstrates that one week after SKPs and collagen co-transplantation, the epithelia were significantly thicker than those treated with collagen sponge only. The wound gaps were completely closed in both SKPs + collagen and collagen only groups, but incomplete wound closure was shown from the control group. By day 14, complete closure of the wound gap could be observed from all 3 groups, which is mainly attributable to keratinocyte migration, fibroblast proliferation and matrix formation. However, no formation of granulation tissue was yet detectable (Fig. 3b). Furthermore, cell infiltration, presumably as a result of keratinocyte migration, keratinocyte proliferation and fibroblast activation, could be observed (Fig. $3 \mathrm{c} \& d$ ). A substantial amount of cells could be observed within the wound gap of the SKPs + collagen group $(207 \pm 53 \%, p<0.01$ vs control; $p<0.01 v s$ collagen only), indicating enhanced fibroblast and keratinocyte activities. A moderate amount of cells was also recorded from the collagen only group $(154 \pm 35 \%, p<$ $0.01 v s$ control), suggesting a beneficial role of collagen sponge in wound closure.

Since collagen formation also represents a crucial stage of wound healing, Masson trichrom staining was employed for wound gap examination. One week after treatment, collagen deposition was present in both SKPs + collagen and collagen only groups. Thicker epidermises were shown from SKPs + collagen and collagen only groups compared to the 


\section{Cellular Physiology \\ Cell Physiol Biochem 2015;37:1725-1737 \\ \begin{tabular}{l|l}
\hline DOI: 10.1159/000438537 & (c) 2015 The Author(s). Published by S. Karger AG, Basel
\end{tabular} and Biochemistry Published online: November 09, 2015 www.karger.com/cpb}

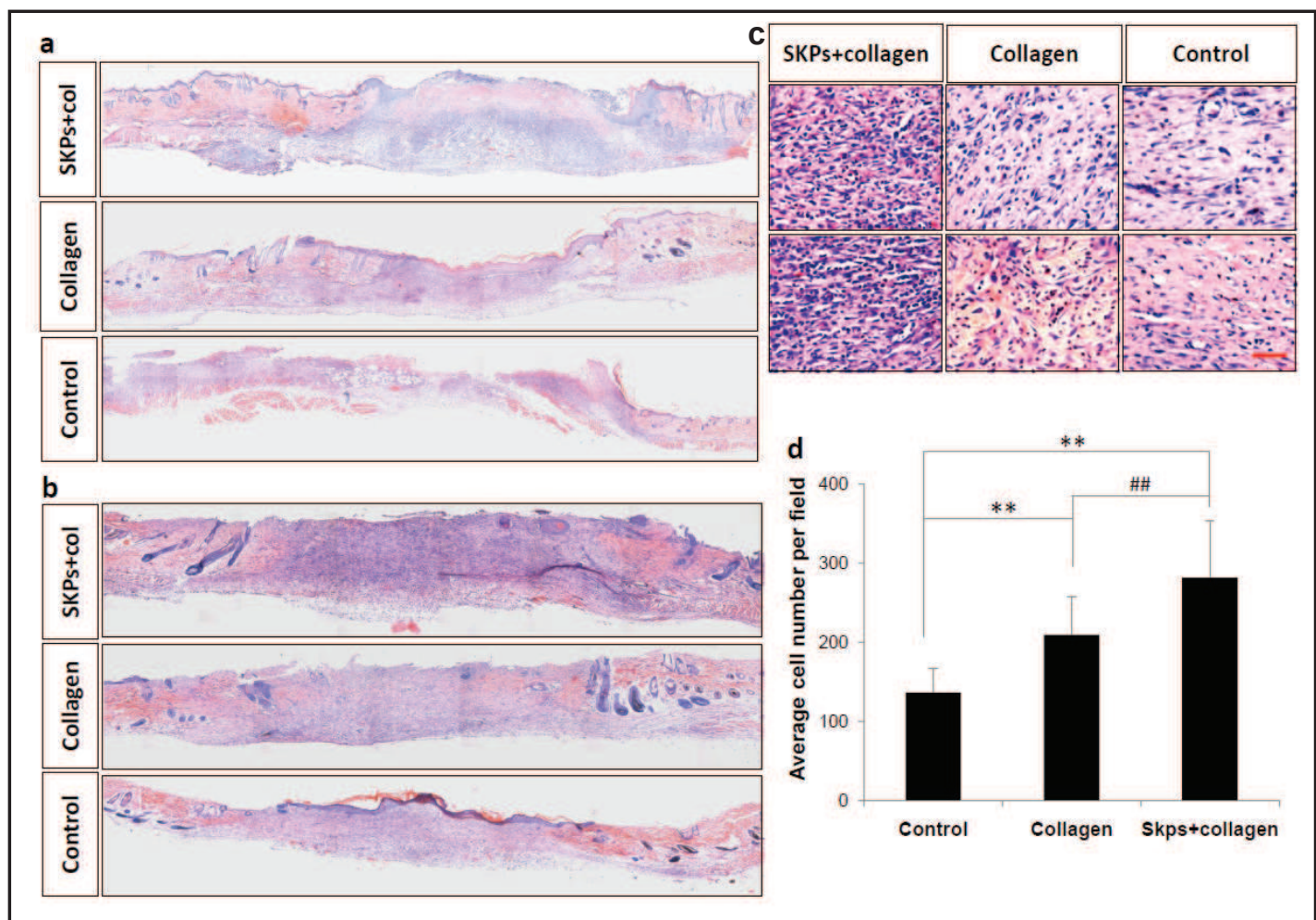

Fig. 3. Effect on epithelial formation of cutaneous wound after treatment with collagen sponge-delivered SKPs or collagen sponge only in T2D mice. (a) Representative images of H\&E staining of epidermises 7 days following treatment with collagen sponge-delivered SKPs (SKPs $+\mathrm{col}$ ) and collagen sponge only (Collagen). (b) Representative images of H\&E staining of epidermises 14 days following treatment with collagen sponge-delivered SKPs (SKPs + col) and collagen sponge (Collagen) only. (c) Representative images showing the base of epidermises obtained from the SKPs + collagen sponge, collagen sponge only and control groups. Scale bar $=50 \mu \mathrm{m}$. (d) Estimated average cell density of epidermises obtained from the SKPs + collagen, collagen sponge only and control groups. Mice with skin abrasion but no treatment at the wounded area were considered as controls. Images are representative of 6 independent experiments. Data are shown as mean \pm standard errors. ${ }^{* *} p<0.01$ compared to control group; \#\# $p<0.01$ compared to collagen sponge only group.

control group, implicating an over-supply of matrix fibres that are necessary for wound gap closure (Fig. 4a). By Day 14, the levels of collagen deposition were comparable between SKPs + collagen and collagen only groups. Increased average cell density was observed within the wound gaps of SKPs + collagen-treated animals (shown as orange-coloured staining in Fig. 4b), suggesting the occurrence of tissue remodelling which in turn demonstrates the presence of a more advanced wound healing stage of the SKPs + collagen/collagen onlytreated animals.

\section{SKPs and collagen sponge transplantation facilitates local capillary regeneration}

It has been reported that SKPs could differentiate into vascular endothelial cells [20] and vascular smooth muscle cells [21], and thereby enhance neoangiogenesis in wound healing. Collagen sponge has also been developed as wound dressing for cutaneous skin injury by promoting tissue regeneration and wound closure $[32,33]$. To elucidate the ways in which SKPs and collagen sponge co-transplantation facilitates wound healing, collagen sponge scaffold with and without SKPs were applied to skin wounds of diabetic mice for 2 weeks. Average capillary density was estimated by immunefluorescence staining for isolectin, which is commonly known as an endothelial marker (Fig. 5a\&c). Substantial increase in capillary density was recorded from the SKPs + collagen group, showing a $222 \pm 74 \%$ elevation over 


\section{Cellular Physiology \\ Cell Physiol Biochem 2015;37:1725-1737 \\ \begin{tabular}{l|l}
\hline DOI: $10.1159 / 000438537$ & C 2015 The Author(s). Published by S. Karger AG, Basel
\end{tabular} and Biochemistry Published online: November 09, 2015 www.karger.com/cpb}
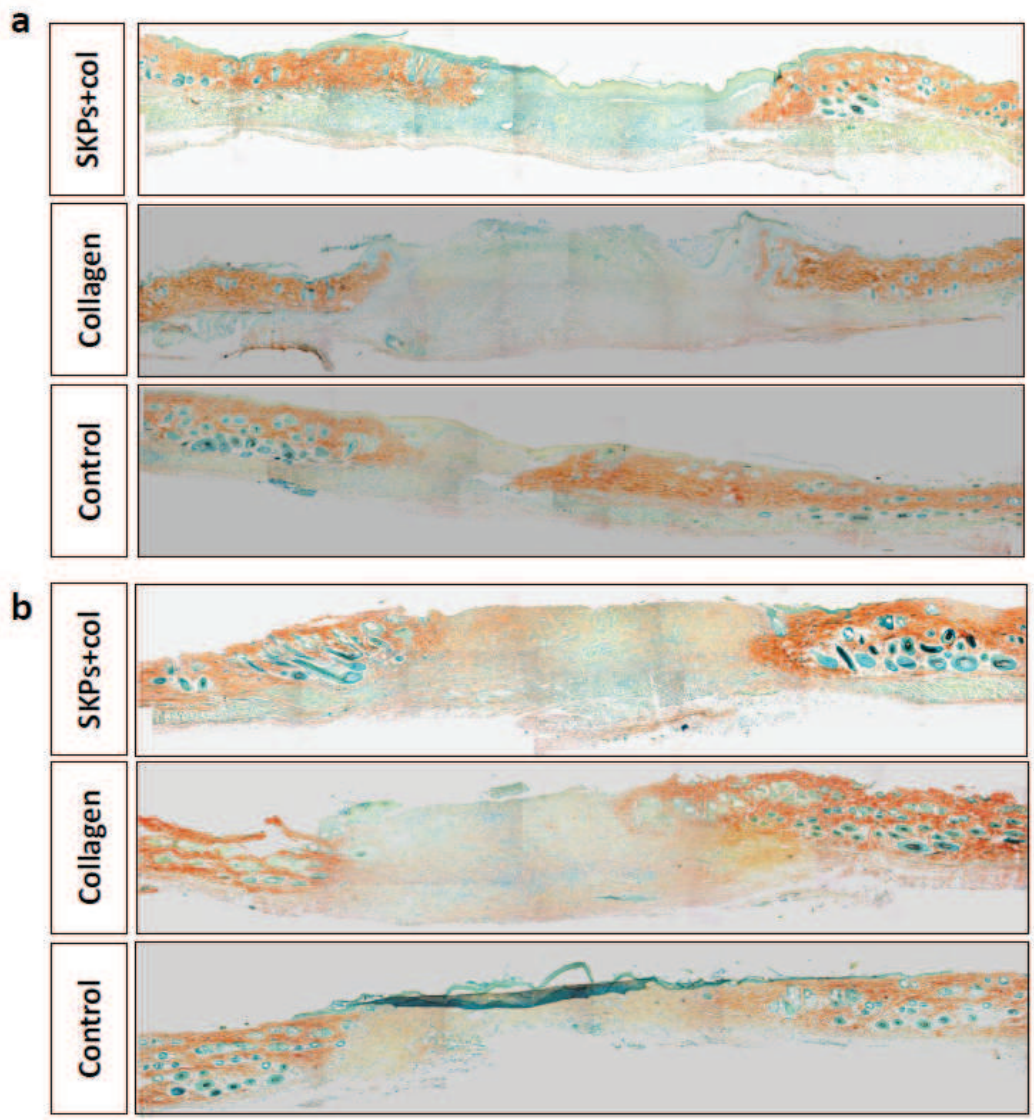

Fig. 4. Effect on collagen deposition and matrix formation of cutaneous wound after treatment with collagen sponge-delivered SKPs or collagen sponge only in T2D mice. (a) Representative images of Masson's trichrom staining of epidermises 7 days following treatment with collagen sponge-delivered SKPs (SKPs + col) and collagen sponge only (Collagen) group. (b). Representative images of Masson's trichrom staining of epidermises 14 days following treatment with collagen sponge-delivered SKPs (SKPs + col) and collagen sponge only (Collagen) group. Mice with skin abrasion but no treatment at the wounded area were considered as controls.

the control group $(p<0.05)$. The collagen only group also exhibited a moderate increase in capillary formation, although it was not statistically significant $(157 \pm 54 \%, p>0.1$ vs control). Similar results were also obtained from $\alpha$-smooth muscle actin ( $\alpha$-SMA) staining, which is often used to identify fibroblasts and vascular smooth muscle cells. For the purpose of the present study however, $\alpha$-SMA was mainly used as one of the vascular markers. As shown in Fig. 5b, fluorescence signals that correspond to $\alpha$-SMA were shown as circular shapes, demonstrating formation of neo-vessels. The SKPs + collagen group exhibited increased average blood vessel density in wound areas compared to controls (163 $\pm 45 \%$, $p<0.05$; Fig. 5d).

SKPs ameliorate cutaneous wound by in vivo differentiation and paracrine signalling

To investigate in vivo SKPs differentiation, SKPs that were used to treat skin wound were pre-labelled with a red fluorescence dye, Dil. Two weeks after SKPs and collagen sponge cotransplantation, immunostaining results revealed overlap of Dil $^{+}$cells and the expressions of $\mathrm{vWF}$ and $\alpha$-SMA, especially at the base of epidermises where keratinocyte migration, fibroblast proliferation and granulation occurs (Fig. 6a). Positive signals of vWF were also found adjacent to Dil $^{+}$cells, demonstrating likely differentiation of the SKPs into vascular 


\section{Cellular Physiology \\ Cell Physiol Biochem 2015;37:1725-1737 \\ \begin{tabular}{l|l}
\hline DOI: 10.1159/000438537 & (c) 2015 The Author(s). Published by S. Karger AG, Base
\end{tabular} and Biochemistry Published online: November 09, 2015 www.karger.com/cpb}

Ke et al.: Skin-Derived Precursors Promote Angiogenesis in Diabetic Wound Healing

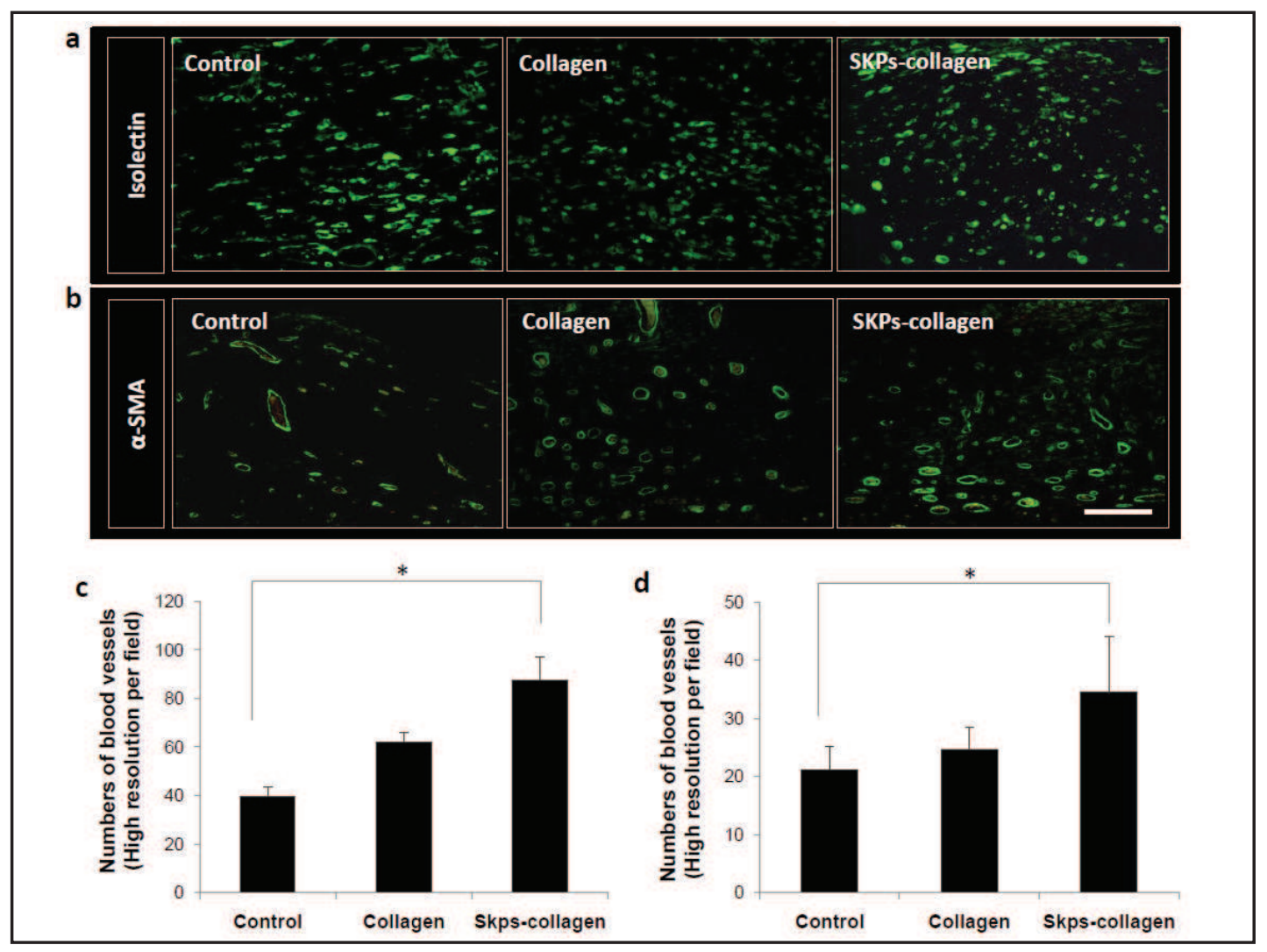

Fig. 5. Collagen sponge-delivered SKPs, but not collagen sponge only, treatment promoted local revascularisation at wound area in T2D mice. (a\&b) Immunohistochemistry staining of $\alpha$-SMA (a) or isolectin (b) reveals increased blood vessel and capillary density, respectively, of wound areas obtained from SKPs + collagen, collagen sponge only and control mice. (c\&d) Average fluorescence density of FITC within the wound area was quantified for isolectin (c) or $\alpha$-SMA (d) and plotted against each treatment group. Images are representative of 6 independent experiments. Scale bar $=50 \mu \mathrm{m}$. Data are shown as mean \pm standard errors.

endothelial and/or smooth muscle cells, both of which are responsible for increased local angiogenesis.

In addition, co-localisation between nestin, a neural stem cell marker, and $\alpha$-SMA, isolectin and vWF was also examined. As shown in Fig. 6b, some co-expression of nestin and $\alpha$-SMA could be observed. Expressions of isolectin and vWF were also detectable adjacent to nestin ${ }^{+}$cells, implicating peripheral axon regeneration parallel to vascular reformation.

\section{Discussion}

Wound healing impairment affects over $50 \%$ of diabetic individuals at some point in their lives, often leads to persistent infection, ulceration and sometimes, more serious complications. As the prevalence of diabetes rises worldwide, non-invasive approaches that could promote wound healing are in urgent need. In recent years, regenerative properties of stem cell transplantation have been reported in multiple organ injuries [7-9, 22, 37]. In particular, protective effects of the SKPs have already been demonstrated against cerebral ischaemia, suggesting a beneficial role of the SKPs in ischaemic injuries [38]. Studies also reported in vivo differentiation of the SKPs into vascular smooth muscle cells, endothelial cells and neurons, all of which are essential components in wound healing and regeneration $[20,21]$. Given the easy accessibility of the SKPs, it has been considered as an ideal candidate for cell therapy against cutaneous wounds [10-12, 20,39]. However, one major drawback 


\section{Cellular Physiology Cell Physiol Biochem 2015;37:1725-1737

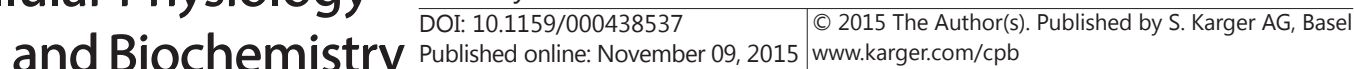

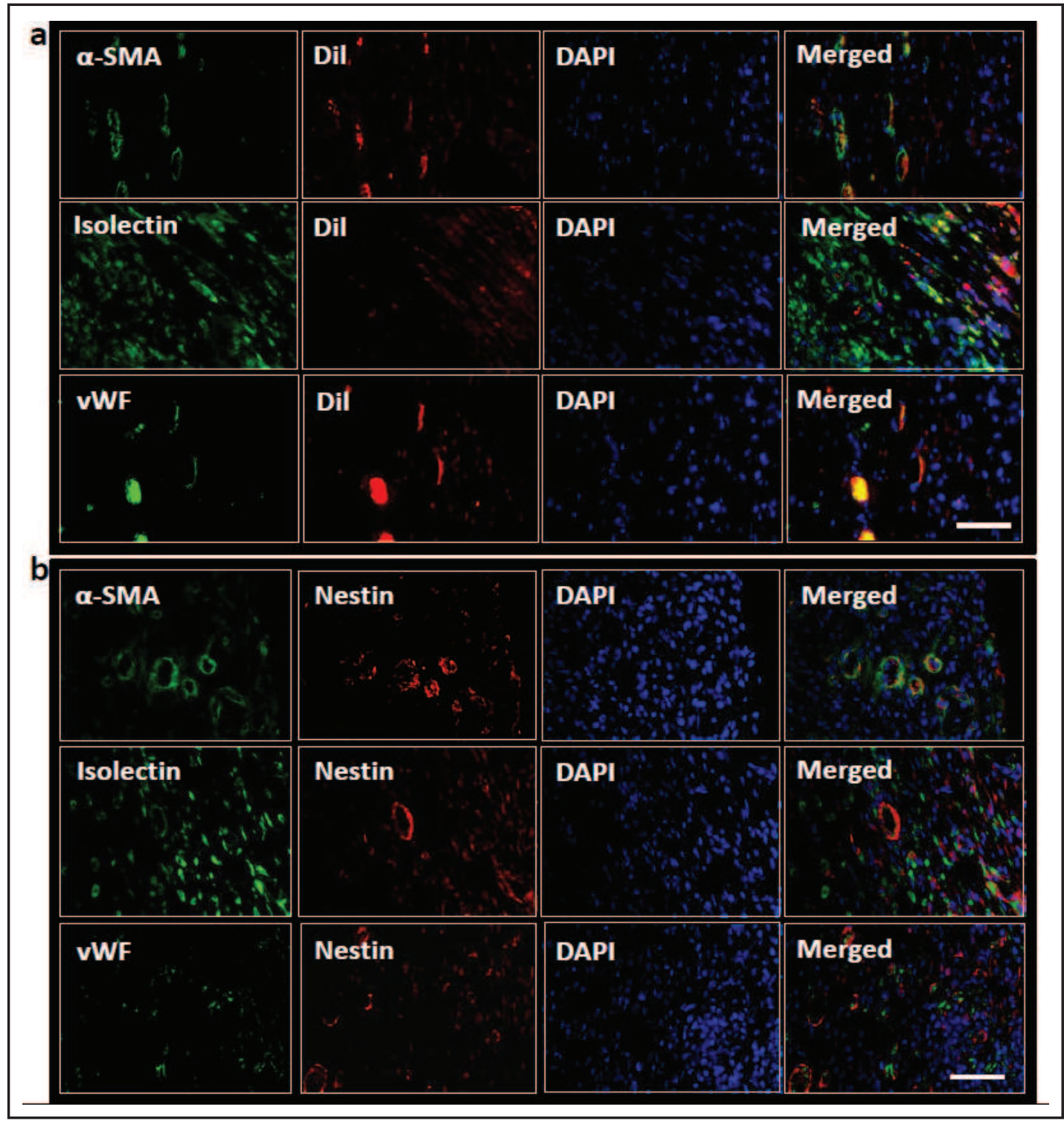

Fig. 6. SKPs promote local angiogenesis via paracrine signalling and direct transdifferentiation into neural and vascular components. (a) Co-expression of Dil ${ }^{+}$cells with $\alpha$-SMA, isolectin and vWF at the base of epidermises surrounding wound gap. Overlapping of $\alpha$-SMA/Dil and vWF and Dil could be observed, demonstrating transdifferentiation of Dil ${ }^{+}$SKPs into fibroblasts, endothelial or vascular smooth muscle cells. Isolectin expression was located adjacent to Dil ${ }^{+}$cells, suggesting paracrine signalling. (b) Double staining of nestin with $\alpha-S M A$, isolectin and vWF. Expressions of isolectin and vWF were found to be adjacent to nestin. Some co-localisation could be observed between $\alpha$-SMA and nestin, indicating neurotrophic effect of the SKPs. Nuclei were stained with DAPI and shown in blue. Images are representative of 6 independent experiments. Scale bar $=50 \mu \mathrm{m}$.

of cell therapy is poor cell survival due to hostile conditions such as hypoxia, ischaemia and local inflammation at the sites of injury [7]. For better post-transplantation SKPs survival, collagen sponge, a natural material that was developed as wound dressing decades ago, was employed in this study as a scaffold for SKPs delivery. Efficacy of SKPs and collagen sponge co-delivery on diabetic wound repair was subsequently examined.

Our initial results showed that despite the lack of statistical significance, SKPs that were co-cultured with collagen sponge exhibited greater cell viability compared to SKPs that cultured in the absence of collagen sponge (Fig. 1). The rate of cell proliferation plateaued KARGER 


\section{Cellular Physiology Cell Physiol Biochem 2015;37:1725-1737 and Biochemistry Published online: November 09, 2015 www.karger.com/cpb \\ Ke et al.: Skin-Derived Precursors Promote Angiogenesis in Diabetic Wound Healing}

after 3 days in culture, during which time the SKPs maintained their original characteristics with low degree of differentiation, suitable for transplantation. For in vivo experiments, dorsal skin of diabetic mice were cut and treated with SKPs and collagen sponge (SKPs + collagen), collagen sponge without SKPs (collagen only) or saline (control). After 14 days, mice treated with SKPs + collagen or collagen only showed more rapid wound healing than the control group (Fig. 2). Histological analyses further revealed better wound gap closure and collagen deposition from both SKPs + collagen and collagen only groups (Fig. 3 a\&b). Despite no apparent differences from histological staining results, elevated average cell densities were observed at the base of epidermises surrounding the wound areas from both SKPs + collagen and collagen only groups (Fig. $3 \mathrm{c} \& d$ ), correspond to increased keratinocyte migration, proliferation as well as fibroblast activities that are crucial for early stage wound healing. Subsequent cell quantification revealed increased average cell density from the collagen only group compared to controls $(154 \pm 35 \%, \mathrm{p}<0.01)$, consistent with its protective property as wound dressing. Indeed, reports showed stimulatory impact of collagen sponge in growth factor activation [27, 32,33], which may explain the pro-proliferative effect observed from the collagen only group. Regarding the SKPs + collagen sponge group, average cell density was substantially higher than the collagen only group $(135 \pm 34 \%, \mathrm{p}<0.01$ vs collagen only group), demonstrating a superior impact of SKPs transplantation in promoting wound closure.

However, the underlying mechanisms via which the SKPs promote cutaneous wound healing remain unknown. In order to identify the ways in which SKPs enhance wound healing, they were pre-labelled with a red fluorescent dye, Dil, before transplantation. As shown in Fig. 5, increased expression of $\alpha$-SMA, one of the vascular markers, could be observed, and statistical significance was only obtained from the SKPs + collagen group. Similarly, fluorescence signals that correspond to capillary endothelial cell marker, isolectin, were also found to be significantly elevated from the SKPs + collagen group. Since no significant impact on local vascular regeneration was recorded from the collagen only group, it could be concluded that the SKPs are mainly responsible for increased local vascular regeneration observed from the SKPs + collage-treated mice. Further staining analyses revealed overlapping between Dil $^{+}$cells and $\alpha$-SMA or vWF, demonstrating direct differentiation of the $\mathrm{Dil}^{+}$SKPs into key components for neoangiogenesis. Expression of isolectin was found to be adjacent to Dil ${ }^{+}$SKPs, suggesting a paracrine effect of the SKPs in vivo (Fig. 6a). Indeed, SKPs could stimulate secretion of growth factors including the vascular endothelial growth factor (VEGF) and basic fibroblast growth factor (bFGF; data not shown), which compensate for reduced growth factor level in chronic diabetic wounds [2]. However, one limitation concerning the use of Dil is its lipophilic property, which enables its labelling ability by binding to the cell membrane. On the other hand, this hydrophobicity also means that Dil could easily transfer from the pre-labelled cells to adjacent cells in vivo, especially over a long period of time, making it a less than ideal approach in such circumstances. As a result, double staining of nestin, a neural precursor marker that is expressed by the SKPs, with $\alpha$-SMA, $\mathrm{vWF}$ or isolectin was also carried out. The results revealed adjacent localisation between nestin and $\alpha$-SMA, isolectin or vWF (Fig. 6b). Furthermore, previous studies have reported that nestin ${ }^{+}$SKPs could differentiate into Schwann cells, which are the major components of the protective myelin sheath surrounding growing axons [13]. Given the positive impact of Schwann cells in axonal reformation following peripheral never injury, the nestin ${ }^{+}$cells that were detected adjacent to vascular markers may be indicative of local nerve remyelination, which in turn implicate a neurotrophic property of the SKPs in promoting peripheral axon elongation in addition to vascular regeneration.

Thus, SKPs delivery accelerated diabetic wound healing mainly by paracrine secretion of pro-angiogenic and neurotrophic factors and direct transdifferentiation into vascular and neural components. Although no apparent difference in wound closure was detectable histologically, better local revascularisation and never regeneration were observed from diabetic mice after SKPs and collagen sponge co-transplantation, emphasizing a superior effect of SKPs therapy in promoting diabetic wound healing. 


\section{Cellular Physiology Cell Physiol Biochem 2015;37:1725-1737 \begin{tabular}{ll|l} 
aOI: 10.1159/000438537 & $\begin{array}{l}\text { C 2015 The Author(s). Published by S. Karger AG, Basel } \\
\text { www.karger.com/cpb }\end{array}$ \\
\hline
\end{tabular} \\ Ke et al.: Skin-Derived Precursors Promote Angiogenesis in Diabetic Wound Healing}

\section{Acknowledgements}

We are grateful for the generous financial support of the National Natural Science Foundation of China (project Nos. 31300732, 31260223, 81260229), the Tianjin Research Programme of Application Foundation and Advanced Technology (project No. 13JCYBJC39300) and the Yunnan Provincial Science and Technology Department Science Foundation (project No. 2012FB044).

\section{Disclosure Statement}

No conflicts of interest are declared by the authors.

\section{References}

1 Braiman-Wiksman L, Solomonik I, Spira R, Tennenbaum T: Novel insights into wound healing sequence of events. Toxicol Pathol 2007;35:767-779.

2 Brem H, Tomic-Canic M: Cellular and molecular basis of wound healing in diabetes. J Clin Invest 2007;117:1219-1222.

3 Immonen JA, Zagon IS, McLaughlin PJ: Topical Naltrexone as Treatment for Type 2 Diabetic Cutaneous Wounds. Adv Wound Care (New Rochelle) 2014;3:419-427.

4 Potter MJ, Banwell P, Baldwin C, Clayton E, Irvine L, Linge C, Grobbelaar AO, Sanders R, Dye JF: In vitro optimisation of topical negative pressure regimens for angiogenesis into synthetic dermal replacements. Burns 2008;34:164-174.

5 Han JW, Choi D, Lee MY, Huh YH, Yoon YS: Bone marrow-derived mesenchymal stem cells improve diabetic neuropathy by direct modulation of both angiogenesis and myelination in peripheral nerves. Cell Transplant DOI:10.3727/096368915X688209.

6 Jia X, Xie X, Feng G, Lu H, Zhao Q, Che Y, Zheng Y, Han Z, Xu Y, Li Z, Kong D: Bone marrow-derived cells can acquire renal stem cells properties and ameliorate ischemia-reperfusion induced acute renal injury. BMC Nephrol 2012;13:105.

7 Ke T, Wu Y, Li L, Liu Y, Yao X, Zhang J, Kong D, Li C: Netrin-1 ameliorates myocardial infarction-induced myocardial injury: mechanisms of action in rats and diabetic mice. Hum Gene Ther 2014;25:787-797.

8 Liu Y, Ye X, Mao L, Cheng Z, Yao X, Jia X, Mao D, Ou L, Li Z, Che Y, Liu N, Steinhoff G, Liu L, Kong D: Transplantation of parthenogenetic embryonic stem cells ameliorates cardiac dysfunction and remodelling after myocardial infarction. Cardiovasc Res 2013;97:208-218.

9 Feng G, Mao D, Che Y, Su W, Wang Y, Xu Y, Fan Y, Zhao H, Kong D, Li Z: The phenotypic fate of bone marrowderived stem cells in acute kidney injury. Cell Physiol Biochem 2013;32:1517-1527.

10 Fernandes KJ, McKenzie IA, Mill P, Smith KM, Akhavan M, Barnabe-Heider F, Biernaskie J, Junek A, Kobayashi NR, Toma JG, Kaplan DR, Labosky PA, Rafuse V, Hui CC, Miller FD: A dermal niche for multipotent adult skin-derived precursor cells. Nat Cell Biol 2004;6:1082-1093.

11 Fernandes KJ, Miller FD: Isolation, expansion, and differentiation of mouse skin-derived precursors. Methods Mol Biol 2009;482:159-170.

12 Toma JG, McKenzie IA, Bagli D, Miller FD: Isolation and characterization of multipotent skin-derived precursors from human skin. Stem Cells 2005;23:727-737.

13 Amoh Y, Li L, Campillo R, Kawahara K, Katsuoka K, Penman S, Hoffman RM: Implanted hair follicle stem cells form Schwann cells that support repair of severed peripheral nerves. Proc Natl Acad Sci U S A 2005;102:17734-17738.

14 Biernaskie JA, McKenzie IA, Toma JG, Miller FD: Isolation of skin-derived precursors (SKPs) and differentiation and enrichment of their Schwann cell progeny. Nat Protoc 2006;1:2803-2812.

15 Guo W, Miao C, Liu S, Qiu Z, Li J, Duan E: Efficient differentiation of insulin-producing cells from skinderived stem cells. Cell Prolif 2009;42:49-62.

16 Mehrabi M, Mansouri K, Hosseinkhani S, Yarani R, Yari K, Bakhtiari M, Mostafaie A: Differentiation of human skin-derived precursor cells into functional islet-like insulin-producing cell clusters. In Vitro Cell Dev Biol Anim 2015;51:595-603. 


\section{Cellular Physiology Cell Physiol Biochem 2015;37:1725-1737 \begin{tabular}{ll|l} 
and Biochemistry $10.1159 / 000438537$ & $\begin{array}{l}\text { O 2015 The Author(s). Published by S. Karger AG, Basel } \\
\text { www.karger.com/cpb }\end{array}$ \\
\cline { 2 - 3 }
\end{tabular} Ke et al.: Skin-Derived Precursors Promote Angiogenesis in Diabetic Wound Healing}

17 Lavoie JF, Biernaskie JA, Chen Y, Bagli D, Alman B, Kaplan DR, Miller FD: Skin-derived precursors differentiate into skeletogenic cell types and contribute to bone repair. Stem Cells Dev 2009;18:893-906.

18 Shu B, Xie JL, Xu YB, Lai W, Huang Y, Mao RX, Liu XS, Qi SH: Effects of skin-derived precursors on wound healing of denervated skin in a nude mouse model. Int J Clin Exp Pathol 2015;8:2660-2669.

19 Hunt DP, Morris PN, Sterling J, Anderson JA, Joannides A, Jahoda C, Compston A, Chandran S: A highly enriched niche of precursor cells with neuronal and glial potential within the hair follicle dermal papilla of adult skin. Stem Cells 2008;26:163-172.

20 Amoh Y, Li L, Yang M, Moossa AR, Katsuoka K, Penman S, Hoffman RM: Nascent blood vessels in the skin arise from nestin-expressing hair-follicle cells. Proc Natl Acad Sci U S A 2004;101:13291-13295.

21 Steinbach SK, El-Mounayri O, DaCosta RS, Frontini MJ, Nong Z, Maeda A, Pickering JG, Miller FD, Husain M: Directed differentiation of skin-derived precursors into functional vascular smooth muscle cells. Arterioscler Thromb Vasc Biol 2011;31:2938-2948.

22 Butler DL, Juncosa-Melvin N, Boivin GP, Galloway MT, Shearn JT, Gooch C, Awad H: Functional tissue engineering for tendon repair: A multidisciplinary strategy using mesenchymal stem cells, bioscaffolds, and mechanical stimulation. J Orthop Res 2008;26:1-9.

23 Wang X, Liu S, Zhao Q Li N, Zhang H, Zhang X, Lei X, Zhao H, Deng Z, Qiao J, Cao Y, Ning L, Duan E: Threedimensional hydrogel scaffolds facilitate in vitro self-renewal of human skin-derived precursors. Acta Biomater 2014;10:3177-3187.

24 Guo B, Lei B, Li P, Ma PX: Functionalized scaffolds to enhance tissue regeneration. Regen Biomater 2015;2:47-57.

25 Molinari C, Rizzi M, Squarzanti DF, Pittarella P, Vacca G, Reno F: 1alpha,25-Dihydroxycholecalciferol (Vitamin D3) induces NO-dependent endothelial cell proliferation and migration in a three-dimensional matrix. Cell Physiol Biochem 2013;31:815-822.

26 Stemberger A, Grimm H, Bader F, Rahn HD, Ascherl R: Local treatment of bone and soft tissue infections with the collagen-gentamicin sponge. Eur J Surg Suppl 1997;578:17-26.

27 Stenzel KH, Miyata T, Rubin AL: Collagen as a biomaterial. Annu Rev Biophys Bioeng 1974;3:231-253.

28 Yang C, Hillas PJ, Baez JA, Nokelainen M, Balan J, Tang J, Spiro R, Polarek JW: The application of recombinant human collagen in tissue engineering. BioDrugs 2004;18:103-119.

29 Geiger M, Li RH, Friess W: Collagen sponges for bone regeneration with rhBMP-2. Adv Drug Deliv Rev 2003;55:1613-1629.

30 Glasgold MJ, Glasgold AI, Silver FH: The use of Collagen Matrix to enhance closure of facial defects. Ear Nose Throat J 1991;70:531-537.

31 Aziz 0, Athanasiou T, Darzi A: Haemostasis using a ready-to-use collagen sponge coated with activated thrombin and fibrinogen. Surg Technol Int 2005;14:35-40.

32 Chvapil M: Considerations on manufacturing principles of a synthetic burn dressing: a review. J Biomed Mater Res 1982;16:245-263.

33 Doillon CJ: Porous collagen sponge wound dressings: in vivo and in vitro studies. J Biomater Appl 1988;2:562-578.

34 Lou S, Gao S, Wang W, Zhang M, Zhang J, Wang C, Li C, Kong D, Zhao Q: Galactose-functionalized multiresponsive nanogels for hepatoma-targeted drug delivery. Nanoscale 2015;7:3137-3146.

35 Lan CC, Wu CS, Huang SM, Wu IH, Chen GS: High-glucose environment enhanced oxidative stress and increased interleukin-8 secretion from keratinocytes: new insights into impaired diabetic wound healing. Diabetes 2013;62:2530-2538.

36 Lim YC, Bhatt MP, Kwon MH, Park D, Na S, Kim YM, Ha KS: Proinsulin C-peptide prevents impaired wound healing by activating angiogenesis in diabetes. J Invest Dermatol 2015;135:269-278.

37 Chen Z, Pradhan S, Liu C, Le LQ: Skin-derived precursors as a source of progenitors for cutaneous nerve regeneration. Stem Cells 2012;30:2261-2270.

38 Mao D, Yao X, Feng G, Yang X, Mao L, Wang X, Ke T, Che Y, Kong D: Skin-derived precursor cells promote angiogenesis and stimulate proliferation of endogenous neural stem cells after cerebral infarction. Biomed Res Int 2015;2015:945846.

39 De Kock J, Meuleman P, Raicevic G, Rodrigues RM, Branson S, Meganathan K, De Boe V, Sachinidis A, LerouxRoels G, Vanhaecke T, Lagneaux L, Rogiers V, Najar M: Human skin-derived precursor cells are poorly immunogenic and modulate the allogeneic immune response. Stem Cells 2014;32:2215-2228. 